\title{
CONSIDERAÇÕES SOBRE UM CASO DE BLASTOMYCOSE \\ PCR
}

FLAVIO A. MAURANO

A opportunidade de ter autopsiado o cadaver de um individuo victimado por blastomy.cose dá-nos motivo para apresentar aigumas considerações a res̃peito dessa entidade morbida que entre nós, infelizmente, é mais commum do que se pensa.

Já sabemos quanto é impreciso o termo "blastomycose" para designar molestias que, embora tenham physionomia clinica mais ou menos identica, são determinadas por agentes os mais variados. Como, porém, as tentativas no sentido de substituir essa denom:nação foram improficuas, em virtude dos conhecimentos ainda inexactos a respeito dos agentes pathogenicos causadores da moles. tia, é preferivel conservarmos o velho termo "blastomycose" para definir seu aspecto clinico e não a sua etiologia.

E' sabido que os agentes da blastomycose comprehendem por um lado os levedos propriamente ditos, e por outro os cogumelos filamentoíos, os quaes pódem dar ás vezes e em certas condições formas de levedos.

Embora o nosso escopo seja considerar o caso sob o ponto de vista clinico e anatomo-pathologico, cumpre-nos, todavia, relativamente á etiologia da blastomycose, citar os agentes descobertos e €studados no Brasil.

Assim, tratando dos saccharomycetaceos, convem lembrarmonos do saccharomyces de Ferrani (Mello, Paes e Souza, 1918) descoberto em abcessos frios multiplos; dos endomycetaceos, o endomyces Cruzi (Mello e Paes, 1917), encontrado num escarro de asthmatico; dos blastoporaceos, a monilia brasiliensis, numa mycose pulmonar mortal (O. de Magalhães, 1918); dos arthrosporaceos, o mycoderma brasiliensis, blastomycoses brasileiras da pelle das mucosas (Splendore); dois blastomycetos incertae sedis, o adenomyces Cruzi (Pedro Dias) encontrado nas lesões da molestia de Hogdkin ou pseudo leucemia e, finalmente, o phialophora verrucosa (Thaxter, Pedroso e Maria Gomes) responsavel pela dermatite verrucosa. Langeron tambem incluiu no grupo dais blastomycoses o sapinho, as anginas cremosas, a lingua negro pilosa, vulvo vaginites, otites, keratites e conjunctivites.

Segundo a localisação, as blastomycowes podem ser cutaneas, mucosas, osseas, visceraes, do tubo digestivo, hepaticas, perito- 
neaes, dos centros nervosos, e tambem blastomycoses generalisadas e dos tumores.

As blastomycoses labiaes 'e buceaes que podem se generalisar e determinar confusão com as leishmanioses das mucosas, são frequentes na America do 'ISul, tendo sido estudadas no extrangeiro por Sommer, Greco, Escomel, e no Brasil par Lutz, Splendore, Carini, Rabello, Miguel Pereira, Montenegro, Pedro Dias, Lindenberg, Terra, W. Machado, 'Gaspar Vianna, Gomes Cruz, Haberfeld, Lordy, I'edroso, Portugal, Aguiar Pupo, Pirajá da Silva e Severiano Magahães. Esta mycose se inicia geralmente na pelle, attingindo mais tarde as mucosas e causando a morte por cachexia e outros factotores. Existe, porém, a forma primaria das mucosas, como se verifica entre nós. Segundo Langeron, as blastomycoses cutaneas, cuja etiologia é muito controversa, podem ser divididas em doís grupos principaes, de accôrdo com os agentes responsaveis:

$\left.1 .^{\circ}\right)$ Dermatite causada pelo myicoderma dermatitis, chamáda dermatite blastomycetica, Molestia de Gilchriāt, Molestia de Chicago, molestia de marcha chronisa, atacando as extremidades, som pouca tendencia a generalisar-se. E' a verdadeira blastomycose cutanea, de prognostico benigno.

$\left.2 .^{\circ}\right)$ Dermatite produzida pelo mycoderma immite, agente este que se confunde com bs coccidios protozoarios, por causa de sua reproducção por endospóros. Pode se generalisar, tornando-se systemica, ou dá metastases nos ganglios (Posadas, Wolbach, Mac Neal, Pedroso).

Os primeiros casos de blastomycoses foram descriptos por Wersicke e Posadas, em. Buenos Áyres, e Giltner, na California. Seguiram-se depois os estudos de Ophüls, Moffit, Gilchrist e outros.

Em 1919, Haberfeld, no Brasil, descreveu o lymphógranuloma ganglionar maligno, de origem blastomycetica, molestia em que ha lesões generalisadas, sendo a principio de localisação ganglionar e provocando a morte. O agente foi chamado "zymonema histosporocellular" que parece ser o mesmo mycoderma immite. Este granuloma é endemico no Brasil, nos Esțados de São Paulo e Minas Geraes, e ataca de preferencia os lavradores de café, tendo sido observado tamibem por Vianna, Pedroso e Magal'hães. Esses agentes são de origem saprophytica. O tratamento pelo iodureto não tem dado resultados. Severiano Magalhães diz, entretanto, ter tido bons resultados com o seu emprego intensivo e prolongado.

Ao lado dessas dermatites ha outras produzida derma, podendo-se-lihes tambem juntar, ao menos provisoriamente, uma forma de dermatite chamada "verrucosa" descoberta em Boston, em 1915, por C. G. Lane e verificada entre nós por Pedroso e J. Maria Gomes. 
Quanto ás blastomycoses generalisadas, ha os estudos americanos de Walker, Montgomery, Ormsby, Hektoen e Hyde. No caso de blastomýcose de Busse-Buschke, o individuo morreu dessa molestia generalisada, com lesões nos olhos, nos ossos e diversas visceras. Outro caso é o de Harter, 'que evoluiu como o de BusseBuschke: - pyolhemia chronica, terminando com a morte. Foram encontradas lesões cerebraes, focos nos figado, pulmões, e gommas sub-cutaneas.

Outras molestias encaixadas por Langeron nas blastomycoses 'internas̃, são a histoplasmose de Darling e a pseudo-leucemia ou molestia de Hogdkin. Quanto a outras localisações de blastomycose, não têm especial interesse.

As manifestações clinicas das blastomycoses são multiplas e rão apresentam uniformiclade. A's vezes trata-se de dermatite, simulando, ora tuberculose, ora leishmaniose, com abcesso miliares, placas verrucosas e destruição intensa das mucosas. As lesõe das mucosas são as mais rebeldé e as mais penosas para os doentes, dando-lhes aspecto repellente e tornando sua vida insupportavel. Quando a lesão das mucosas é primaria ou secundaria á localisação cutanea ou outra, a molestia pode ser considerada incuravel.

A morte nas blastomycoses pode ser attribuida a tres ordens de causas:

a) generalisação: a morte dá-se por pyohemia; b) mo'estias intercurrenteá, principalmente a tuberculose; c) phenomenos mechanicos: edema da glotte, como no nosso caso, que passamos a descrever.

No dia 12 de Março de 1924 procedemos á autopsia de um individuo que fallecera no dia anterior de blastomycose. Tratava-se de Raymundo José Quirino, com 36 annos de tlade, de nacionalidade brasileira, de rasa parda, casado, lavrador, procedente de Catanduva, e que deu entrada na 4." Enfermaria de Medicina de Homens, serviço do professor A. Lindenberg, onde, do seu relatorio que nos foi cedido gentilmente pelos drs. Nicoláu Rossetti e Abilio de 'Castro, consta o seguinte:

ANAMNESE: Homem alto, de esqueleto bem desenvolvido, dá a impressão de ter sido um individuo robusto. Actualmente de aspecto edemaciado, enfraquecido e tristonho. A anamnese familiar nada revela de interessante. Anteriormente á molestia actual o paciente só soffreu maleita.

Ha quatro annos teve uma inflammação da garganta $\epsilon$ de então para cá o mal foi-sie alastrando lentamente, invadindo a mucosa da bocca, debilitando-lhe o organismo e abatendo-lhe o morai Diz o doente: "Eu era um homem espirituoso, até o espirito perdi" 
ESTADO ACTUAL: Examinando-se a mucosa da cavidade buccal, nota-se actualmente uma vasta ulceração de profundidade văriavel, mas em geral superficial, borcos irregulares, um pouco salientıs. occupando toda a face interna do labio inferior, parte das bochechas em correspondencia, e logo atraz das commissuras labiaes, dorso e base da lingua. A ponta da lingua, entumecida e deformada, é excavada por ulcerações mais profundas. 0 fundo das lesões, especialmente o das dos labios e das bochechas, é de côr branco-opalina e recoberto de granulações pequenissimas que lie emprestam um aspecto de todo caracteristico: como se esse fundo branco-opalino fosse picado de pequenissimos pontos de côr variante entre o amarello claro e o vermello escuro. Fira dos bordos, invadindo os tecidos limitrophes, notam-se microabcessos ligeiramente salientes, de côr amarello-clara e do tamanho de uma ponta a uma cabeça de alfinete. A ulceração limita-se, na semi-mucosa do labio inferior, por um bordo bem determinado, saliente e polycyclico. A salivação é abundantissima. Na região maxillar, á direita, apalpa-se uma massa irregular, bem delímitada, bosselada, não deslocavel, constituida por ganglió̃ infarctados.

o exame microscopico de um fragmento da lingua, retirado por biopsia, revelou que a mucosa we apresenta hypertrophiada, emquanto o chorion é tomado por processo inflammatorio intenso, representado por lymphocytos, plasmocytos, e alguns eosinophilos e neutrophilos. De permeio a isso tudo vêem-se cellulas de protoplasma ligeiramente claro, nucleo redondo, central ou excentrico e de coloração rosea. São os blastomycetos. (Relatorio da peça (biopsia) N.॰ 1.281, do laboratorio Anatomo-Pathologico da Santa Casa de Misericordia de São Paulo).

A autopsia, procedida no Instituto Anatomo-Pathologico da Faculdade de Medicina de São Paulo, sob a direcção do Prof. Robert A. Lambert) pelo Dr. João Montenegró e nós, revelou as seguintes lesões:

Blastomycose do labio, gengivas, lingua, pharynge e larynge Edema da glotte

Infarctamento dos ganglios mediastinicos e sub-maxillares

Infarctamento das glandulas sub-maxillares

Peri-splenite chronica

Varizes da mucosa rectal

Infiltração gordurosa do figado

Ancylostomiase

Caries dentarias

Adherencias pleureaes direitas

Tuberculose pulmonar (ligeira) 


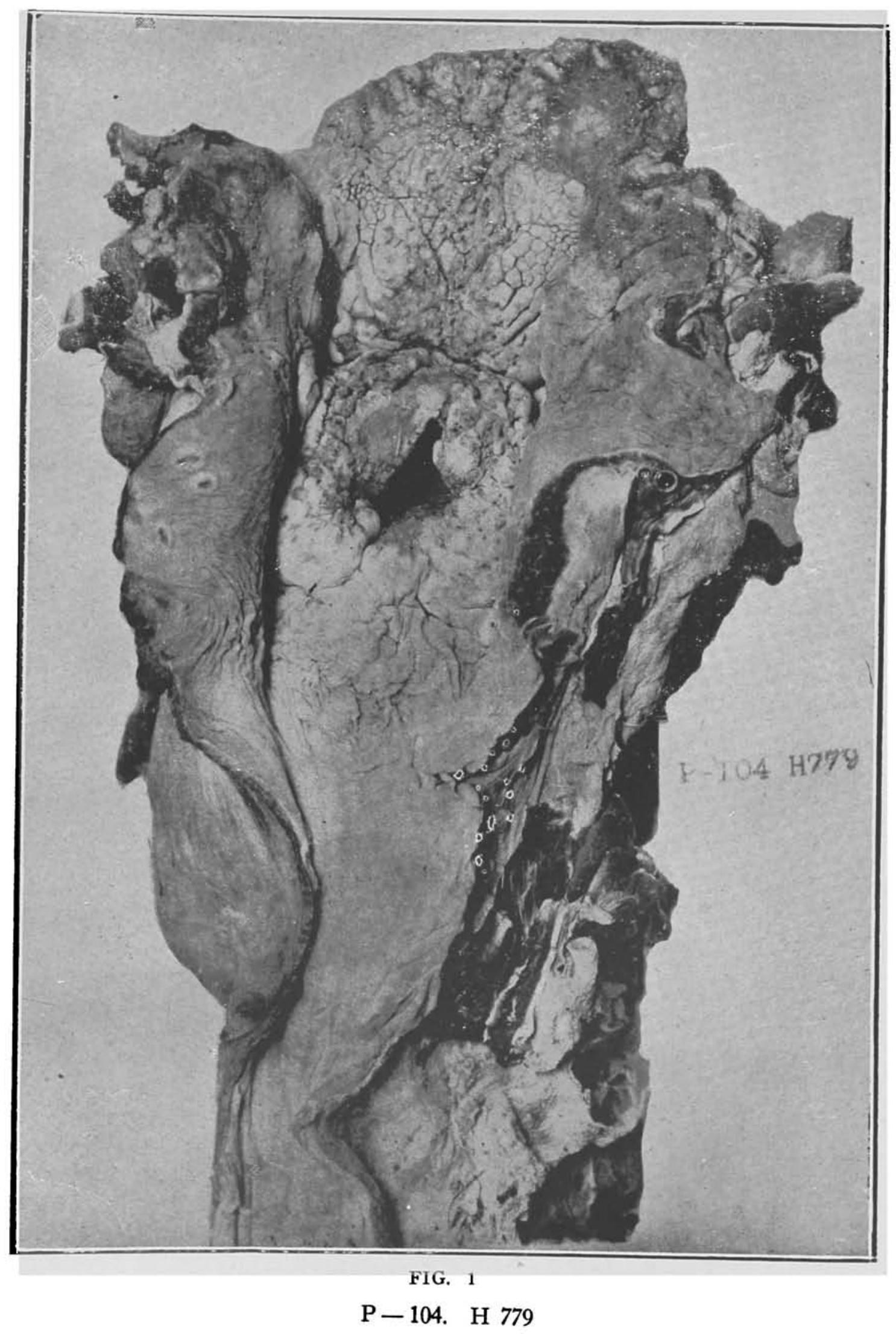





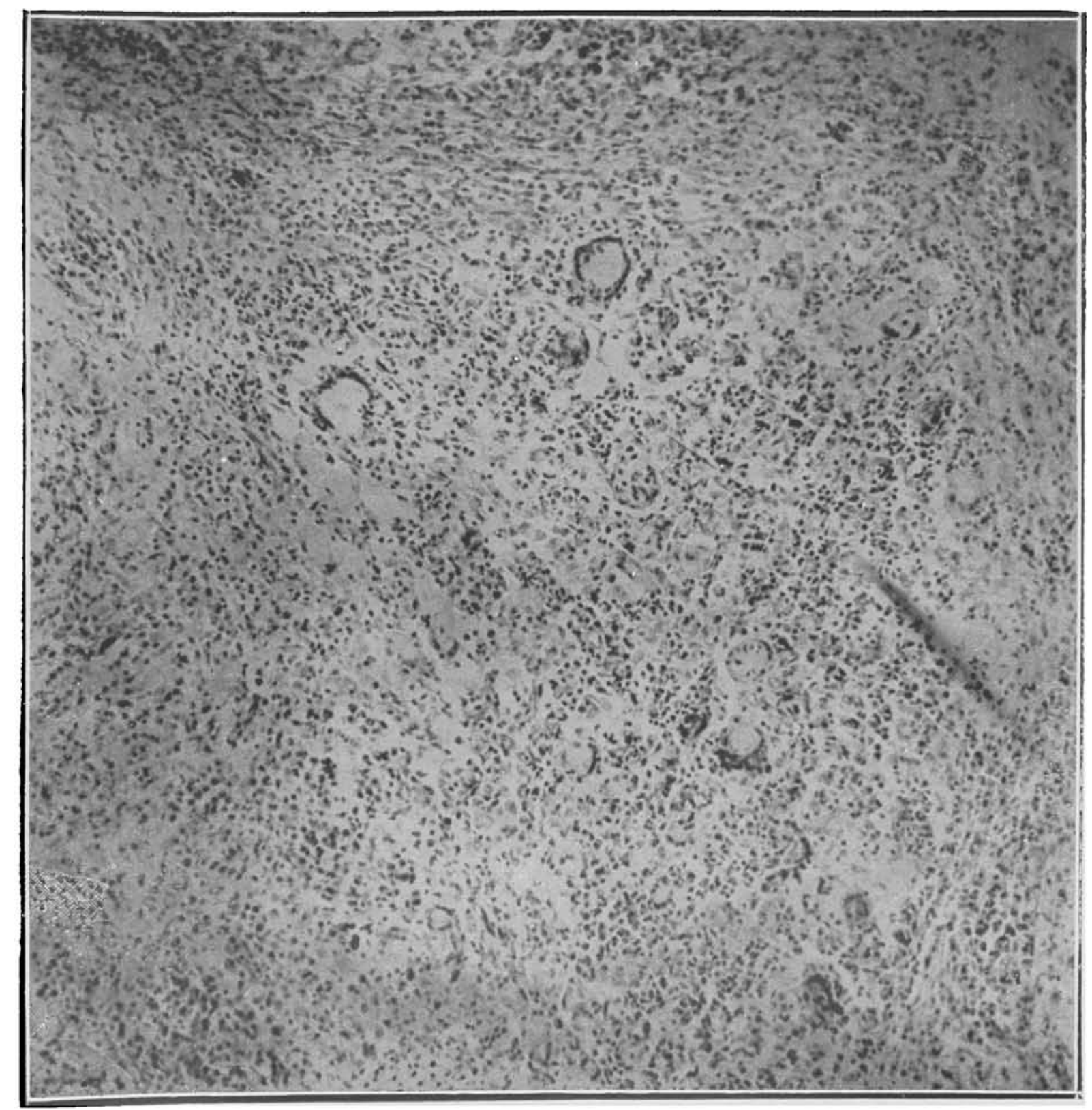

FIG. 2

MP 133 A. 779.24 Object. $16 \mathrm{~mm}$ Ocul. $5 \mathrm{Z} 200$ Diam. 

Os exames microscopicos de fragmentos de orgãos retirados, não accusaram outro fóco de blastomycose nas visceras.

As lesões do labio, gengivas, lingua, pharynge e larynge foram diagnosticadas microscopicamente: granuloma. (Vide Fig. n. ${ }^{\circ}$ ) os cortes mostravam intenso infiltrato constituido por lymphocytos, plasmazellen, cellulas endotheliaes e cellulas gigantes.

Provavelmente a morte, no nosiso caso, deu-se por edema da glotte.

E' um caso em que se constata a localisação primaria nas mucosas: a predileçãa pela larynge, pharynge, asisoalho da bocca e o estabelecimento duma molestia intercurrente, que é a tuberculose.

Fig. N.॰ 1 - Notar a extensão da ulceração que da base da lingua se estende ao pharynge, larynge, destruindo a epiglotte. A glotte acha-se edemaciada (P. 104 H. 779).

Fig. N.o 2 - Mostra uma microphotographia dum corte de lingua corado pela hematoxy'ina-eosina. Notar o grande infiltrato que constitue o granuloma. Vêem-se no corte diversos gigantosytos. MP. 133 A. 779-24. Object. $16 \mathrm{~mm}$. Ocul. $5 \mathrm{Z} 200$ Diam.

\section{BIBLIOGRAPHIA}

DIVERSOS AUTORES: The Mycosis. Oxford Medicine. V. 5, p. 3:97.

LANGERON - Les Blastomyeoses. Nouveau Traité de Medicine, par G. H.Roger, Widal et Teissier. Fascieule 4.

W. OSLER - Modern Medieine. V. 1, p. 346.

W HAWES e P. F. MORSE - Report of two cases of blastomycosis. Boston Med. \& Surg.; 1915, V. 185, p. 315.

DOZ-EEN - Blastomyeosis in Perú. Journ. of Amer. Med. Assoc., 1921, V. 76, p. 622 .

M. HAASE, E. R. HALL AND C. H. MARSHALL - Report of a ease of local blastomyeosis. Journ. of Amer. Med. Assoc., 1922, v. 78,

L. GRAVES - Systemic Blastomycosis. Amer. Journ. Trop. Med., 1922 , V. 2 , p. 123-132.

O. DA FONSEICA - Sobre os agentes das blastomyeoses européas, cyclo sexuado e posição systematica do levedo de Hudelo. Brazil Medico, 1922, v. 36, p. 2, 101.

F. TERRA - Tres Casos de Blastomycose. Brazil Medico, 1923, Vol. 37 , p. $4-44$.

F. LUGDEN - A case of Blastomycosis. British Medical Journal, 1923. V. 2, p. 78-81.

LEITÃO DA CUNHA - Estruetura e operabilidade dos blastomas. Archivos Brasileiros de Medicina, 1921, V. 11, p. 391-429. 